\title{
Arcobacter butzleri and A. cryaerophilus in human, animals and food sources, in southern Chile
}

\author{
Heriberto Fernandez ${ }^{1}$, Maria Paz Villanueva ${ }^{1}$, Ingrid Mansilla ${ }^{2}$, \\ Mario Gonzalez ${ }^{1}$, Fadua Latif ${ }^{1}$ \\ ${ }^{1}$ Instituto de Microbiologia Clinica, Universidad Austral de Chile, Valdivia, Chile. \\ ${ }^{2}$ Instituto de Salud Pública de Chile, Santiago, Chile
}

Submitted: February 1, 2014; Approved: June 6, 2014.

\begin{abstract}
The isolation frequency of Arcobacter species in children with diarrhea, fowls, mammals and food of avian and marine origin was established. In all the samples it was possible to isolate Arcobacter species corresponding 201 (39.4\%) to A. butzleri and 24 (4.7) to A. cryaerophilus. Both species were simultaneously isolated in $19(3.7 \%)$ being $A$. butzleri the most frequently isolated species.
\end{abstract}

Key words: Arcobacter diarrhea, reservoirs, food.

\section{Introduction}

Arcobacter sp. belongs to Campylobactereaceae family as was proposed by Vandamme et al. (1991). This genus included 18 species with A. butzleri, A. cryaerophilus and A. skirrowii been considered the only emerging enteropathogens of relevance for animals and humans (Lehner et al., 2005; Wesley and Miller, 2010; Collado and Figueras, 2011; Euzéby, 2014). A. skirrowii has been isolated from sheep and cattle with diarrhea, aborted pig fetus, and chronic and acute diarrhea in humans. A. cryaerophilus and A.butzleri have been associated with abortion and enteritis in animals as well as with diarrhea and bacteremia in children and adults. The transmission of these organisms occurred by fecal-oral, waterborne, by food of animal origin or by direct contact with animals (Lehner et al., 2005; Wesley and Miller, 2010; Collado and Figueras, 2011).

In Chile there is scarce information regarding this group of bacteria. Fernández et al. (2004) reported the first isolates in this country. Due to the clinical relevance of Arcobacter as foodborne pathogen and the need to get more information related to its ecological distribution and prevalence, we present this communication. We are reporting the prevalence of Arcobacter in children with diarrhea, in animal reservoirs and in food of animal origin. The study was performed in the south part of Chile (Los Rios Region, $39^{\circ} 16^{\prime}$ and $40^{\circ} 41^{\prime}$ latitude South and $71^{\circ} 35^{\prime}$ longitude West until the Pacific Ocean).

\section{Material and Methods}

We studied a total of 510 samples. The type and origin of the samples are described in Table 1. Human feces (approx. $2 \mathrm{~g}$ ) were inoculated into Arcobacter broth $(20 \mathrm{~mL})$ and food samples (approx. $25 \mathrm{~g}$ previously vortex) were added into $125 \mathrm{~mL}$ of Arcobacter broth (Houf et al., 2001). After $48 \mathrm{~h}$ of incubation at $30^{\circ} \mathrm{C}$ under aerobic conditions, 100 microliters aliquots were streaked onto Arcobacter agar plates (Houf et al., 2001). Additionally, 400 microliters of enrichment broth were filtrated onto blood agar plates using a $0.45 \mathrm{~m} \mu$ filter (Fernández et al., 2004). All the plates were incubated under the same conditions described above. Colonies with 2 to $4 \mathrm{~mm}$ diameter and smooth borders were tested for Gram stain, wet preparation for contrast microscopy, and oxidase and catalase tests. Those colonies with oxidase and catalase positive of rods gram negative with characteristic motility spinning around their long axes under the contrast microscope were considered presumptively Arcobacter sp.. Those colonies were streaked in blood agar plates for confirmatory testing using multiplex PCR as proposed by Houf et al. (2000) for the confirmation of the three main species of Arcobacter. 
Table 1 - Prevalence of Arcobacter species among human, animal and food samples.

\begin{tabular}{|c|c|c|c|c|c|c|c|c|}
\hline \multirow[t]{2}{*}{ Origin } & \multirow[t]{2}{*}{ Type } & \multirow[t]{2}{*}{ Number } & \multicolumn{2}{|c|}{ A. butzleri } & \multicolumn{2}{|c|}{ A. cryaerophilus } & \multicolumn{2}{|c|}{ A. butzleri + A. cryaerophilus } \\
\hline & & & $\mathrm{N}^{\circ}$ & $\%$ & $\mathrm{~N}^{\circ}$ & $\%$ & $\mathrm{~N}^{\circ}$ & $\%$ \\
\hline Children & Feces & 83 & 3 & 3.6 & 0 & 0 & 0 & 0 \\
\hline Pig & Feces & 135 & 55 & 40.7 & 13 & 9.6 & 12 & 8.9 \\
\hline Bovine & Feces & 75 & 20 & 26.7 & 5 & 6.7 & 3 & 4.0 \\
\hline Chicken & Feces & 20 & 2 & 10.7 & 4 & 20.0 & 0 & 0 \\
\hline Chicken & Meat & 125 & 90 & 72.0 & 2 & 1.6 & 3 & 2.4 \\
\hline Chicken & Stomach & 25 & 8 & 32.0 & 0 & 0 & 0 & 0 \\
\hline Chicken & Liver & 25 & 18 & 72.0 & 0 & 0 & 1 & 4.0 \\
\hline Seafood & Mussels & 22 & 5 & 22.7 & 0 & 0 & 0 & 0 \\
\hline Total & & 510 & 201 & 39.4 & 24 & 4.7 & 19 & 3.7 \\
\hline
\end{tabular}

\section{Results}

As indicated on Table 1, Arcobacter was isolated from all the sample types. A total of 201 (39.4\%) strains of A. butzleri, and $24(4.7 \%)$ of $A$. cryaerophilus were isolated. In $19(3.7 \%)$ of the samples studied both species were recovered simultaneously.

From children with diarrhea and from mussels, only A. butzleri was isolated (3.6\% and $22.7 \%$, respectively).

In chicken feces, $A$. cryaerophilus was most frequently isolated (20\%) than A. butzleri $(10 \%)$ but in pig and bovine feces occurred the opposite. The frequency of $A$. butzleri was $40.7 \%$ in pigs and $26.7 \%$ in bovines while for A. cryaerophylus they were 9.6 and $6.7 \%$, respectively.

High frequencies of Arcobacter were found in poultry meat ready for human consumption (chicken meat $92 \%$, muscular stomach $32 \%$, and liver $92 \%$ ).

\section{Discussion}

A. butzleri has been described as the Arcobacter species more frequently isolated from clinical and environmental specimens, as well as from food of animal origin (Lehner et al., 2005; Wesley and Miller, 2010; Collado and Figueras, 2011), and it was confirmed as the most prevalent specie in this study. A. butzleri was the only Arcobacter specie isolated from children with diarrhea with $3.6 \%$ prevalence. The prevalence of Arcobacter in this study was higher to the prevalence found by Prouzet-Mauleon et al. (2006) in France (1\%) and to the prevalence previously reported by Collado et al. (2013) in Valdivia, Chile (1.7\%). However, the prevalence of Arcobacter in this study was similar to the prevalence observed by Vandenberg et al. (2004) in Belgium (3.5\%). Similar to the reports of Prouzet-Mauleon et al. (2006) and Collado et al. (2013), we were unable to recovery $A$. skirrowii or A. cryaerophilus from feces of children with diarrhea. The only study in which $A$. cryaerophilus was isolated from feces of children with diarrhea was the report of Vandenberg et al. (2004) but as in other studies included the present one, A. skirrowii was not recovered.

In Europe, the reports indicated that Arcobacter species represent the $4^{\text {th }}$ place in frequency of isolation among the member of the Campylobactereaceae family in samples of fecal origin (Vandenberg et al., 2004; Prouzet-Mauleon et al., 2006). The high prevalence of $A$. butzleri found in diarrhea, similarly at is occurs with Campylobacter jejuni/coli, might be an indicator of similar risk factors in the environment (Wesley and Miller, 2010, Fernández, 2011).

In chicken feces, A. butzleri was isolated (10\%) as well as $A$. cryaerophilus (20\%). The prevalence found in this study is similar to previous studies (Fernández et al., 2007; Houf, 2010). However, the prevalence of Arcobacter species in pig and bovine feces was lower that the prevalence of Campylobacter species in the same type of samples (Table 1). The findings in this study were similar to results previously reported in the bovine and pig samples (Fernández, 2011; Fernández et al., 2007; Houf, 2010).

Some investigators have proposed that the low prevalence of Arcobacter species in chickens is an indicator that this organism is not a natural intestinal commensal in chicken and represent only a transient organism incapable to colonize the gut chicken. A potential explanation for this phenomenon is that the normal corporal temperature in chicken $\left(40.5\right.$ to $\left.42{ }^{\circ} \mathrm{C}\right)$ represents a limiting factor for the colonization of chicken with Arcobacter species because their optimal growth temperature range is from 26 to $30^{\circ} \mathrm{C}$, contrasting with Campylobacter that is recovered in high prevalence in chicken due to its optimal growth temperature that is around $42^{\circ} \mathrm{C}$ (Wesley and Miller, 2010; Houf, 2010). However, in contrast from the animal samples, the recovery of Arcobacter from poultry meat ready for human consumption is high ( $92 \%$ in chicken meat, 32\% in muscular stomach, and $92 \%$ in liver). These results of prevalence of Arcobacter in poultry meat are in agreement with those previously reported (Fernández et al., 2001; Collado and Figueras, 2011). According to Wesley and Miller (2010) 
and Houf (2010), the explanation for this discrepancy is that poultry may be contaminated in processing plants and the temperature used for storage of the product $\left(4^{\circ} \mathrm{C}\right.$ and/or room temperature) may favor the colonization by external sources.

The frequency of recovery Arcobacter species was higher in feces from bovine, pigs, and poultry meat samples. Among those samples, A. butzleri and A. cryaerophilus were recovered simultaneously. This observation has been previously reported (Lehner et al., 2005; Collado and Figueras, 2011; Houf, 2010).

The prevalence of $A$. butzleri in mussels was $22.7 \%$, this prevalence was different from previous reports by Fernandez et al. (2001) and Collado et al. (2009) who reported higher prevalence of A. butzleri and the recovery of additional Arcobacter species in the same type of seafood.

In summary, this report confirmed the presence of Arcobacter species in children with diarrhea in the south region of Chile. In addition, this report confirmed the presence of Arcobacter in animal reservoirs and in food of animal origin such as poultry meat and seafood. We believe that the presence of Arcobacter may be an indicator of fecal contamination in the human environment and food supplies. Therefore, it is imperative to establish a constant survey to determine its environmental distribution, its presence in other animal sources as well as the epidemiological relationship between Arcobacter strains isolated from different sources and their mode of transmission. This information will contribute to clarify and a better understanding of the epidemiology of this emerging enteropathogen.

\section{Acknowledgments}

The study was financially support by projects DIDUACH S-2004-01 and FONDECYT 1110202. Thanks are due to Dr. Guillermo Pérez-Pérez (New York University Langone Medical Center) for their critical review and scientific advice.

\section{References}

Collado L, Figueras MJ (2011) Taxonomy, epidemiology, and clinical relevance of the genus Arcobacter. Clin Microbiol Rev 24:174-192.

Collado L, Guarro J, Figueras MJ (2009) Prevalence of Arcobacter in meat and shellfish. J Food Protect 72:11021106.
Collado L, Gutiérrez M, González M et al. (2013) Assessment of the prevalence and diversity of emergent campylobacteria in human stool samples using a combination of traditional and molecular methods. Diag Microbiol Infect Dis 75:434-436.

Euzéby JP (2014) List of Prokaryotic names with Standing in Nomenclature - Genus Arcobacter. Available at: http://www.bacterio.cict.fr/a/arcobacter.html. Accessed March 15, 2014.

Fernández H, Krause S, Villanueva MP (2004) Arcobacter butzleri an emerging enteropathogen: communication of two cases with chronic diarrhea. Braz J Microbiol 35:216218.

Fernández H, Otth L, Wilson M et al. (2001) Occurrence of Arcobacter sp. in river water, mussels and commercial chicken livers in Southern Chile. Int J Med Microbiol 291:140.

Fernández H, Vera F, Villanueva MP (2007) Arcobacter and Campylobacter species in birds and mammals from Southern Chile. Arch Med Vet 39:163-165.

Fernández H (2011) Campylobacter and campylobacteriosis: a view from South America. Rev Peru Med Exp Salud Publica 28:121-127.

Houf K, Devriese LA, De Zutter L et al. (2001) Development of a new protocol for the isolation and quantification of Arcobacter species from poultry products. Int $\mathrm{J}$ Food Microbiol 71:189-196.

Houf K, Tutenel A, De Zutter L et al. (2000) Development of a multiplex PCR assay for the simultaneous detection and identification of Arcobacter butzleri, Arcobacter cryaerophilus and Arcobacter skirrowii. FEMS Microbiol Lett 193:89-94.

Houf K (2010) Arcobacter In: Dongyou L. (ed) Molecular Detection of Foodborne Pathogens. CRC Press, Boca Raton, p. 283-298.

Lehner A, Tasara T, Stephan R (2005) Relevant aspects of Arcobacter spp. as potential foodborne pathogen. Int J Food Microbiol 102:127-135.

Prouzet-Mauleon V, Labadi L, Bouges N et al. (2006) Arcobacter butzleri: Underestimated enteropathogen. Emerg Infect Dis 12:307-309.

Vandamme P, Falsen E, Rossau R et al. (1991) Revision of Campylobacter, Helicobacter and Wolinella taxonomy: emendation of generic descriptions and proposal of Arcobacter gen. nov. Int J Syst Bacteriol 41:88-103.

Vandenberg O, Dediste A, Houf K et al. (2004) Arcobacter species in humans. Emerg Infect Dis 10:1863-1867.

Wesley IV, Miller GW (2010) Arcobacter: an opportunistic human food-borne pathogen? In: Scheld WM, Grayson ML, Hughes JM (ed) Emerging Infections 9. ASM Press, Washington D.C., p. 185-211.

\section{Associate Editor: Nilton Erbet Lincopan Huenuman}

All the content of the journal, except where otherwise noted, is licensed under a Creative Commons License CC BY-NC. 